\title{
CD38 and Regulation of the Immune Response Cells in Cancer
}

\author{
Sanyog Dwivedi $\mathbb{D}^{1,2}$ Erika P. Rendón-Huerta $\mathbb{D}^{1},{ }^{1}$ Vianney Ortiz-Navarrete $\mathbb{D}^{2}{ }^{2}$ \\ and Luis F. Montaño $\mathbb{D D}^{1}$ \\ ${ }^{1}$ Laboratorio Inmunobiología, Departamento Biología Celular y Tisular, Facultad de Medicina, UNAM, Mexico \\ ${ }^{2}$ Departamento de Biomedicina Molecular, Centro de Investigación y Estudios Avanzados, IPN, Cinvestav, Mexico \\ Correspondence should be addressed to Vianney Ortiz-Navarrete; vortiz@cinvestav.mx and Luis F. Montaño; lfmontmx@ \\ yahoo.com
}

Received 23 December 2020; Revised 12 February 2021; Accepted 17 February 2021; Published 27 February 2021

Academic Editor: Nicola Silvestris

Copyright (c) 2021 Sanyog Dwivedi et al. This is an open access article distributed under the Creative Commons Attribution License, which permits unrestricted use, distribution, and reproduction in any medium, provided the original work is properly cited.

\begin{abstract}
Cancer is a leading cause of death worldwide. Understanding the functional mechanisms associated with metabolic reprogramming, which is a typical feature of cancer cells, is key to effective therapy. CD38, primarily a NAD + glycohydrolase and ADPR cyclase, is a multifunctional transmembrane protein whose abnormal overexpression in a variety of tumor types is associated with cancer progression. It is linked to VEGFR2 mediated angiogenesis and immune suppression as it favors the recruitment of suppressive immune cells like Tregs and myeloid-derived suppressor cells, thus helping immune escape. CD38 is expressed in M1 macrophages and in neutrophil and T cell-mediated immune response and is associated with IFN $\gamma$-mediated suppressor activity of immune responses. Targeting CD38 with anti-CD38 monoclonal antibodies in hematological malignancies has shown excellent results. Bearing that in mind, targeting CD38 in other nonhematological cancer types, especially carcinomas, which are of epithelial origin with specific anti-CD38 antibodies alone or in combination with immunomodulatory drugs, is an interesting option that deserves profound consideration.
\end{abstract}

\section{Introduction}

Cancer immunotherapy has progressed enormously since the identification of immune checkpoints. The list of putative stimulatory and inhibitory checkpoints so far clearly established is extensive: ICOS/ICOS-L, GTR/GTR-L, CD27/ CD70, CD40/CD40-L, DNAM-1/CD155, MHC-II/LAG-3, PD1-L1/2, Galectin-9/TIM-3, and many more [1]. The search for new immune checkpoint targets is now centered towards the adenosinergic pathway and its metabolite adenosine (ADO), that support immune suppression within the tumor microenvironment (TME) $[2,3]$ as it limits the functionality of T, Dendritic, and NK cells, as well as macrophages and neutrophils [4].

The accumulation of adenosine in the TME is partly dependent on two ectoenzymes entangled in canonical and noncanonical pathways [5]. One is the ectonucleoside triphosphate diphosphohydrolase (NTPDase, CD39) that converts ATP released by cell lysis or by exocytosis of ATP- containing vesicles via transport vesicles or via lysosomes into AMP, and the other is ecto-5-nucleotidase (Ecto5" $\mathrm{N}$ Tase, CD73) that dephosphorylates AMP into adenosine [6]. The generation of adenosine by a noncanonical pathway begins with NAD in a reaction run by the multifunctional transmembrane protein $\mathrm{CD} 38$ [7].

CD38 has come into consideration as its involvement in adenosine-mediated immunosuppression within the tumor microenvironment has been established [8]. CD38 is a multifunctional ectoenzyme that functions as a nicotinamide adenine dinucleotide $(\mathrm{NAD}+)$ glycohydrolase and catalyzes the synthesis and degradation of cADPR affecting calcium signaling and release, thus decreasing extracellular $\mathrm{NAD}+$, altering calcium cascade and deeply contributing to adenosine-mediated immune suppression (Figure 1), which alters the activity of $\mathrm{T}, \mathrm{NK}$, and dendritic cells and attracts migration of suppressor cells like MDSCs, Tregs, and Bregs [9-11]. The effect upon immune cells is mainly via modulation of FasL expression [12-14]. Alterations in CD38/FasL 
regulated apoptosis have been reported in myeloma $[15,16]$ and in NK cells of gastric cancer patients $[17,18]$. We have recently gathered evidence that suggests that the overexpression of some tight junction proteins in gastric cancer cells affects CD38-related FasL expression and activity on NK cells. Our aim was to emphasize the role of CD38 on the immune suppression of some malignant neoplasms and to emphasize its role as an interesting target for cancer immunotherapy [19].

1.1. CD38 Structure. CD38 is a $45 \mathrm{kDa}$ type II transmembrane glycoprotein with a single transmembrane segment near its N-terminus. It shares a 20-30\% sequence identity with Aplysia ADP-ribosyl cyclase, BST-1, also termed CD157, and a GPIanchored protein found in Schistosoma mansoni. It is formed by two identical monomers that favor a physiologically stable structure with a pocket at the middle of the cleft, that is, the enzyme active site. The crystal structure of the extramembrane domain, which is fully active enzymatically and is crystallized as head-to-tail dimers, has been well determined [20, 21]. It is expressed in high densities on plasma cells, plasmablasts, natural killer cells, plasmacytoid dendritic cells, and activated B and $\mathrm{T}$ lymphocytes in healthy subjects and in hematological tumors including multiple myeloma [22].

1.2. CD 38 Function. CD38 functions as a lymphocyte receptor and transducer of signals and an ectoenzyme that generates cyclic adenosine diphosphate-ribose involved in intracellular calcium mobilization (Figure 1). First thought to be expressed only on thymocytes and activated $\mathrm{T}$ cells, CD38 was later found to be widely expressed on B cells, circulating monocytes, dendritic cells, granulocytes, plasma cells, both resting and circulating NK cells, neutrophils, and granulocytes. CD38 is also found on the surface of erythrocytes and platelets, where it plays an essential role, together with platelet/endothelial cell adhesion molecule 1 (CD31), in the microenvironment retention of cancer cells [22]. CD38 is also expressed in the cytoplasm and nucleus of nonlymphoid cells such as normal prostatic epithelial cells, pancreatic islet cells, smooth and striated muscle cells, renal tubules, retinal gangliar cells, and cornea.

As a surface receptor, CD38 is necessary for the activation and proliferation of immune cells. Its IFN $\gamma$ and TNF $\alpha$ induce its expression in macrophages and dendritic cells [23]. It establishes a weak and dynamic interaction with the nonsubstrate ligand CD31, in an interaction necessary for leukocyte adhesion and migration. CD38 has a very small cytoplasmic tail suggesting it is unable to initiate a signaling cascade and so it associates with other signaling receptors such as TCR/CD3 in T cells, BCR (CD19/CD21) in B cells, and CD16/CD61 in NK cells. In addition, CD38 ligation with a counter ligand induces the expression and secretion of IL$1 \beta$, IL-6, IL-10, and IFN $\gamma$ from monocytes and $\mathrm{T}$ cells. NAADP, produced through the enzymatic activity of CD38 [24], regulates $\mathrm{T}$ cell activation, proliferation, and chemotaxis.

CD38 is found in recycling endosomes that contain perforin and granzymes in the immunological synapse when the TCR of cytotoxic T cells is engaged. CD38 is expressed on membrane rafts where it promotes cell signaling via $\mathrm{AKT}$ and ERK activation and it is exported out of the cells through the exocytic pathway. CD38 association with the signaling complex CD16/CD61 in the NK cell membrane has a critical role in transducing activating signals. $\mathrm{CD} 38^{\text {high }} \mathrm{CD} 8^{+} \mathrm{T}$ cells suppress the proliferation of $\mathrm{CD} 38^{-} \mathrm{CD} 4^{+} \mathrm{T}$ cells [25], thus indicating its capacity to modulate $\mathrm{T}$ cell subsets with regulatory properties. CD38 signaling upon ligation induces IL$1 \beta$, IL-6, and IL-10 secretion and enhanced IL- 12 production in synergy with IFN $\gamma$ in dendritic cells $[26,27]$.

High CD38 expression in immune cells such as T regs, B regs, MDSCs, and CD16-CD56 + NK cells contribute to a change in their immune function [28-30]. A typical example of the latter is represented by the CD4+CD2 $5^{\text {high }}$ FOX3+ Treg cells with high CD38 expression that define a suppressive subset of Tregs in multiple myeloma and nonHodgkin lymphoma via cytokine dependent mechanisms. However, CD31- Tregs depicted reduced immune suppressive activity that indicates the importance of CD38/ CD31 interaction in Treg mediated immunosuppression [31]. CD38 ${ }^{\text {high }} \mathrm{B}$ reg cells produce IL-10, which inhibits $\mathrm{T}$ naïve cell differentiation to Th1 and Th17 cells while supporting the proliferation of $\mathrm{T}$ regs $[32,33]$. The immunosuppressive role of myeloid-derived suppressor cells (MDSCs) is strongly expanded in the cancer microenvironment [29], which is well documented. CD38 expression is considered as a marker of MDSCs activity and CD38 ${ }^{\text {high }}$ MDSCs have more prominent immune suppressive effects. At the same time, MDSCs promote neovascularization and tumor invasion (Figure 2).

1.3. CD38 and the Tumor Microenvironment. Tumor microenvironment (TME), a coordinated network of immune, nonimmune, and cancer cells with other noncellular components, is vital for the development, progression, immune suppression, and persistence of cancer [35] as biological processes such as hypoxia, angiogenesis, autophagy, apoptosis resistance, and metabolic reprogramming are triggered. The enhanced concentration of adenosine in the TME leads to an increase or decrease of adenylate cyclase or intracellular cyclic adenosine monophosphate in immune cells expressing adenosine receptors ( $T$ cells, NK cells, dendritic cells, neutrophils, macrophages), thus interfering with the activation of immune cells and favoring tumor progression [36, 37]. The accumulation of adenosine within the TME causes immune suppression; targeting CD38 enzymatic activity would largely influence tumor cells. Moreover, targeting CD38 will result in an accumulation of $\mathrm{NAD}+$ that is by itself a danger signal.

TME is also characterized by the presence of hypoxia due to poor blood supply and increased oxygen consumption. $\mathrm{NAD}+$ is produced by the salvage pathway in hypoxic TME, which is further converted to adenosine by CD38-expressing cells, thus further suppressing the immune response by recruitment of MDSCs, Tregs, tumor associated macrophages (TAMs) $[38,39]$. Besides ADO arbitrated immune suppression, CD38 bestowed NAADP is also involved in 


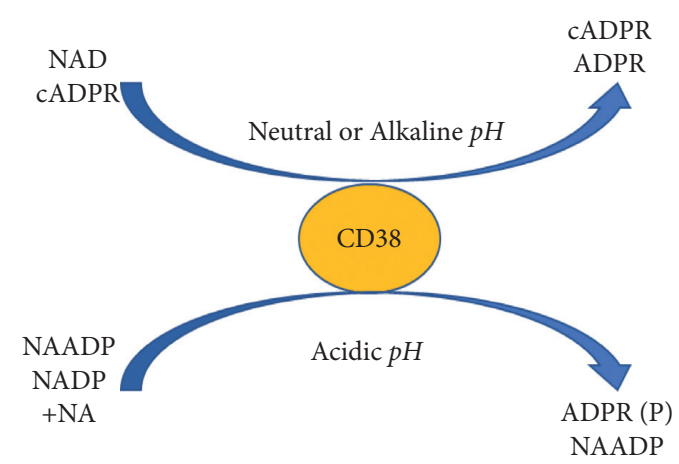

(a)

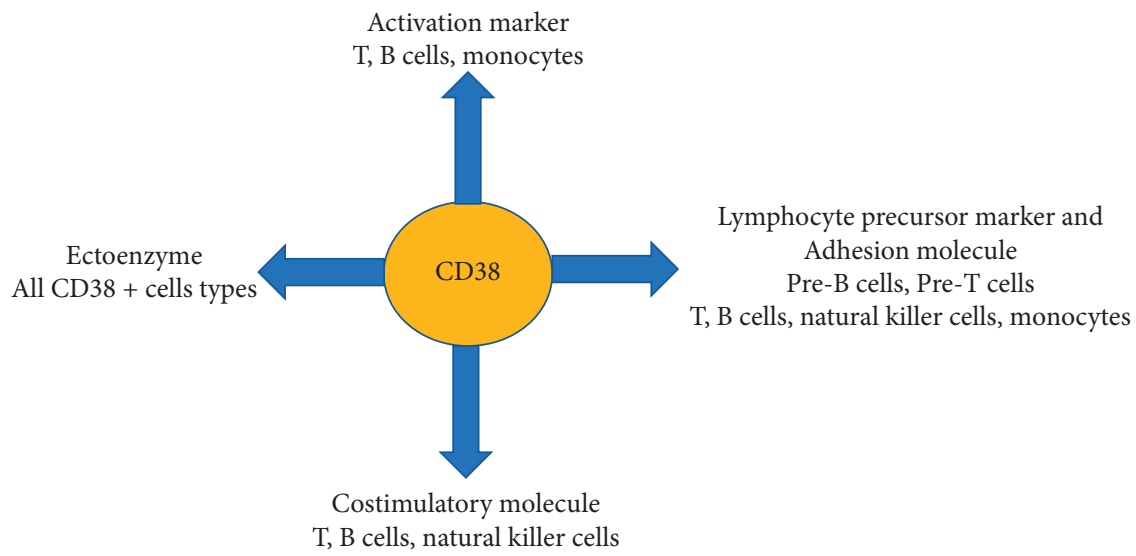

(b)

Figure 1: (a) Enzymatic activity of CD38 in different pH. (b) Various cell-mediated functions. Abbreviations used: cyclicADP-ribose, cADPR; ADP-ribose, ADPR; nicotinic acid adenine dinucleotide phosphate, NAADP; nicotinic acid, NA; ADP-ribose-2'-phosphate, ADPRP.

VEGF mediated angiogenesis through its involvement in $\mathrm{Ca}+$ signaling. VEGF interacts with receptors VEGFR1 and VEGFR2. VEGF binding to VEGFR2 leads to the release of $\mathrm{Ca}++$ in a process where CD38 contributes [40-43]. Therefore, cells overexpressing CD38 in the TME direct the generation of an immune suppressive environment that reduces effector $\mathrm{T}$ cell functions but also promotes angiogenesis provides immune escape and helps in cancer progression. For instance, in chronic lymphocytic leukemia $\mathrm{CD} 38+$ clones in the TME have a survival advantage over CD38- clones as they depict higher migration towards the chemokine CXCL12 resulting in enhanced homing to lymphoid tissue and improved survival with higher expression of VEGF and antiapoptotic protein Mcl1[44]. Metabolic reprogramming of $\mathrm{NAD}^{+}$regulation via inhibition of CD38 has been proposed as a strategy for improving the efficacy of immune-based therapies and appears to play a significant role in the regulation of metabolism and immunomodulation of the tumor microenvironment [28, 45-47].

1.4. CD38 in Multiple Myeloma. Multiple myeloma is a type of cancer where malignant plasma cells overexpressing CD38 accumulate in the bone marrow. Interestingly, within the bone marrow microenvironment, myeloma cells are protected to CD38 antibody-induced cellular cytotoxicity by upregulating the expression of antiapoptotic molecules such as survivin [48]. Additionally, CD38 increases the phosphorylation of PI3K, AKT, and mTORC and upregulates the PI3K/AKT/mTOR pathway, which is related to metabolic reprogramming and proliferation of cancer cells, while such effects were significantly reversed with mTOR inhibitor, rapamycin $[49,50]$. Indeed, $\mathrm{mTOR}$ and RICTOR are overexpressed in MM endothelial cells while mTORC2 and its downstream effectors are linked with an angiogenic switch to MM [51]. This suggests inhibition of the PI3K/AKT/mTOR pathway with a dual mTOR inhibitor along with anti-CD38 therapies will exhibit a synergistic effect in CD38expressing MM cells [51].

In this environment, the use of anti-CD38 mAbs depletes CD38+ MDSCs, T regs, and B regs immune suppressive cells and enhances antitumor activity $[52,53]$, but as anti-CD38 mAbs downregulates CD38 expression in tumor cells, immune escape, and disease progression is favored [54]. Histone deacetylase (HDAC) inhibitors (Panobinostat, Ricolinostat) upregulate CD38 RNA levels and CD38 surface expression on multiple myeloma cells [55], thus disrupting latency of the malignant cells. Interestingly, an all-trans retinoic acid (ATRA) combined with anti-CD38 treatment 
was able to reduce complement inhibitor proteins CD55 and CD59 expression on anti-CD38 resistant cells and improved complement-mediated cytotoxicity [56]. Ricolinostat decreases phosphorylation of AKT and mTOR downstream molecules, upregulates the expression of the Th1 transcription factor T-Bet, and decreases the suppressive function of Treg cells [57]. A significant decrease in IL-10 and Foxp3 in Tregs and improved proliferation and function of CD4+CD25-T cells and CD8+ and NK cytotoxic cells has been observed when an anti-CD38 monoclonal antibody was combined with lenalidomide, an immunomodulator that increases Th1 cytokine production and stimulates clonal $\mathrm{T}$ cell proliferation and NK cell activity [58]. STAT3, an oncogenic protein, has been targeted with a novel formulation of nanoparticles packaged with STAT 3 inhibitor linked with anti-CD38 mAb that improved uptake by MM cell lines; these nanoparticles depicted a 4-fold reduction in tumor size compared with nanoparticles carrying STAT3 inhibitor only [59].

1.5. CD38 in Epithelial Cancers. In esophagus cancer, tumorderived signals such as IL-6, IGFBP3, and CXCL16 trigger the expansion of monocytic MDSCs with increased CD38 expression. This CD38+ MDSC population is halted in the early stages of differentiation, which express elevated levels of iNOS and increased activation of NFKB, resulting to be more potent in suppressing T cells in the TME. In CD38+ cervical cancer cells, the PI3K/AKT pathway and its downstream target gene p53 expression are upregulated $[49,60]$. In gastric cancer, a CD19+CD24hiCD38hi B reg population that plays immunosuppressive roles by producing IL-10 and TGF $\beta$ has been identified; this cell population is inhibiting cytokine production by $\mathrm{CD} 4+\mathrm{T}$ cells and promotes the conversion of CD4 + CD25- effector T cells to $\mathrm{CD} 4+$ FoxP3+Treg cells, which collectively promote tumor growth [30].

High CD38 expression has been observed in hepatic carcinoma TME and in tumor infiltrating lymphocytes (TILs). However, CD38 + TILs provide antitumor responses through secretion of cytotoxic compounds and inflammatory cytokines [61, 62]. These CD38+ TILs secrete high levels of IFN $\gamma$ and in combination with Sorafenib, a kinase inhibitor used for advanced cancer [63], patients' survival improves notably [64]. There is a CD38+ M1 macrophage population in TME that produces high levels of IL-6 and $\mathrm{TNF} \alpha$ with concomitant CD80 expression, prompts more inflammation and helps in tumor suppression [63]. CD38 also serves as a coreceptor in MHC-II mediated T cell activation [65]. Increased CD38 expression induces immunosuppressive effects via its adenosinergic activity and can also cause resistance to anti-PD1/PDL1 treatment in HCC patients [66]. These results reinforce, understanding variability in CD38 expression of TILs in TME may improve the effectiveness of anti-PD1 immunotherapy with a suitable anti-CD38 agent.

Studies performed with CRISPR/Cas9-based knockout of CD38 in A549 adenocarcinoma human alveolar basal epithelial cell line exhibited inhibition of anchorage- independent cell growth, cell invasion, and xenograft growth in nude mice. This is consistent with results obtained with lung cancer cell lines and patient specimens that show increased levels of CD38 mRNA and protein expression of CD38 [67]. Blocking CD38 led to a significant decrease in T regs within TME of an in vivo mouse model of lung cancer. CD38 is also involved in the control of nonsmall cell lung cancers (NSCLS), which cover $85 \%$ of lung cancers [68]. NSCLS treatment was centered on platinum-based chemotherapy followed by cytotoxic chemotherapy, but recently agents targeting CTLA- 4 and PD- 1 pathways have been included. Unfortunately, this treatment frequently develops resistance mediated by Interferon $\beta$ and all-trans retinoic acid after a mean 5 -week period, but Interestingly CD38+ CD8 $\mathrm{T}$ cells proliferation was induced after antiPDL-1 therapy [69]. This CD38+ T cell population corresponds to early activated and nonexhausted effector cells [70]. The strong correlation between CD38 and inflammation in the TME supports the idea of combining antiCD38 therapy with existing anti-PD-1/PDL-1 treatments.

Gliomas, frequent malignant brain tumors, respond poorly to conventional and recently developed cytotoxic chemotherapy. Nevertheless studies performed in CD38 deficient mice showed attenuated tumor size, progression, and improved life expectancy [71]. The glioma TME plays an important role in tumor progression [72]. Tumor associated microglia/macrophages (TMM), formed by a small proportion of resident brain $\mathrm{CD} 38+$ microglia and infiltrating monocytes, constitute $40 \%$ of the tumor [73, 74]. These TMMs secrete IL-1, basic fibroblast growth factor, VEGF, and regulate $\mathrm{Ca}++$ mobilization via $\mathrm{CD} 38$ mediated cADPR, contribute to TMM activation, angiogenesis $[75,76]$, and immunosuppression, thus helping in tumor progression $[77,78]$.

In melanoma anti-PD-1 and anti-CTLA- 4 based immunotherapies improve survival significantly in advanced cases $[79,80]$ while targeting CD38 in melanoma TME could provide synergistic effects. In mouse models, inhibition of CD38 restricted primary tumor growth and was associated with lower rates of pulmonary and brain metastasis as a result of promoted cell death, reduction in cancer-associated fibroblast, and prevention of angiogenesis. Targeting CD38 mediated NAADP synthesis, which is responsible for neoangiogenesis and Ca++ signaling, with Ned-19, an NAADP inhibitor, constrained melanoma growth, vascularization, and metastasis [42]. Thus, reinforcing the importance of CD38 mediated NAADP inhibition in the activity of tumorpromoting components of the TME. An effect of CD38 inhibition is the reduction of adenosine in the melanoma TME that results in the inhibition of antitumor responses. Fortunately the addition of adenosine in primary melanoma cell lines restores the proliferation of CD4+ and CD8+ T cells $[38,81]$.

1.6. Anti-CD38 Agents and Their Therapeutic Use. Daratumumab is a human specific IgG1 anti-CD38 approved as a single agent or in combination regimens for the treatment of relapsed/refractory multiple myeloma $[82,83]$. 


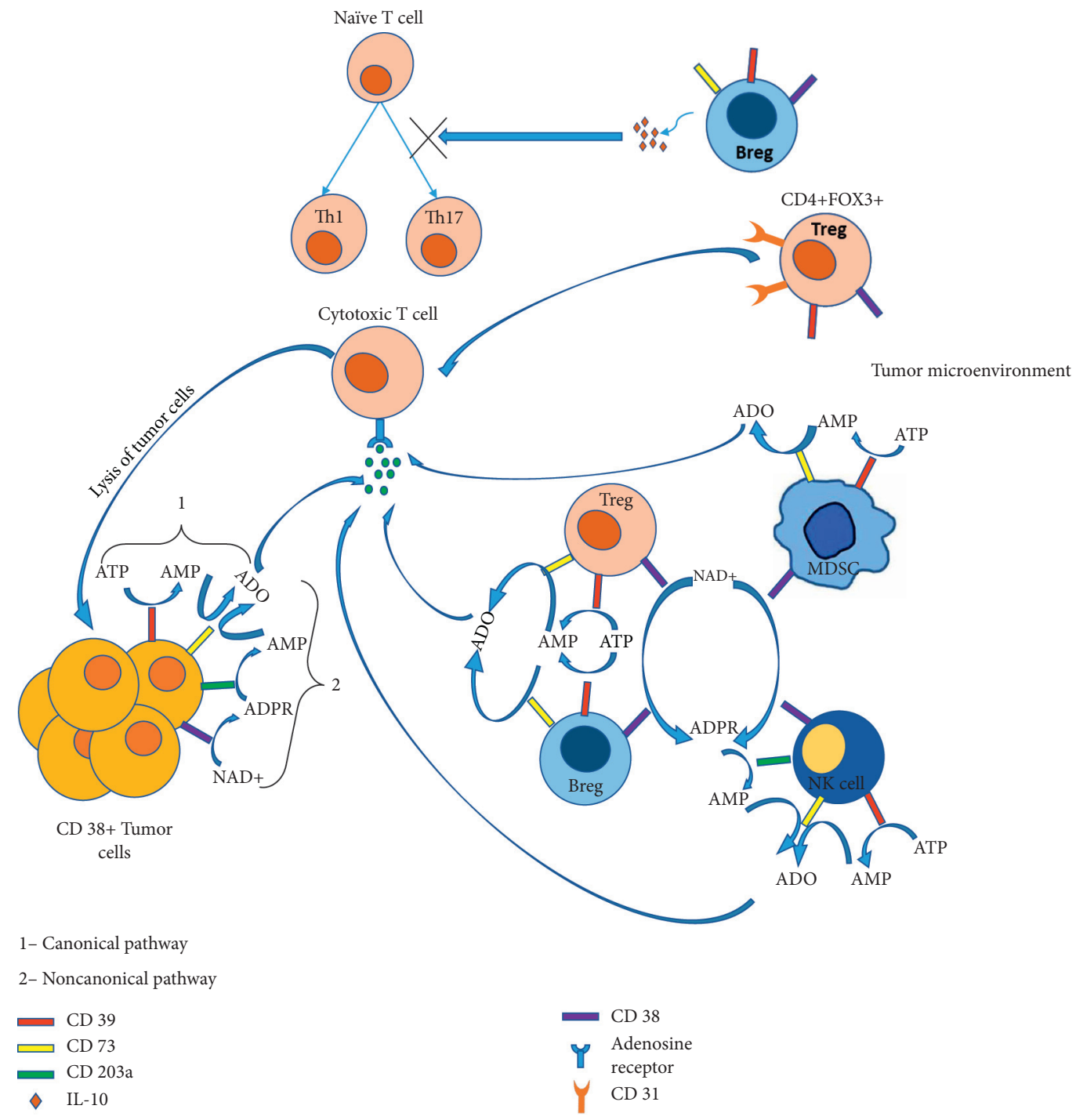

Figure 2: CD38 positive immune suppressive cell types; T regs, Bregs, specific Nk cell type, and tumor cells in TME produce adenosine (ADO) resulting suppression of cytotoxic activity effector $\mathrm{T}$ cells. CD31 $+\mathrm{T}$ regs contribute to immune suppression with an unknown mechanism, while Bregs prompt IL-10 mediated inhibition of naïve T cell differentiation into Th1 and Th17 but promote Treg proliferation. It has also been established that senesce drives the expression of CD38 in macrophages and endothelial cells [34]. CD38 in activated NK cells upregulates the release of IFN $\gamma$ and TNF $\alpha$ and promotes degranulation, albeit depletion of CD38+ NK cells within the tumor does not correlate with patient response to antiCD38 treatment.

It triggers ADCC, CDC, and TAM in CD38+ multiple myeloma cells in both sensitive and drug resistance patients, modulates the enzymatic activity of CD38, and reduces adenosine levels $[84,85]$. Daratumumab combined with dexamethasone and a proteasome inhibitor (Velcade) or a $\mathrm{TNF} \alpha$ inhibitor (Revlimid) has been approved in patients with at least one previous line of therapy. A study on newly diagnosed transplant ineligible patients compared with the gold-standard bortezomib-Melphalan-Prednisone regimen with or without Daratumumab and found that the latter increased significantly the overall response rate and improved complete response and progression-free survival [86]. A similar improvement has been determined in newly diagnosed multiple myeloma and transplant-eligible patients treated with Daratumumab and the standard Velcade-
Thalidomide-Dexamethasone regime [87]. Clinical trials with Daratumumab alone or in combination with hematological and nonhematological malignancies are in progress (Table 1) (Figure 3).

Daratumumab reduces suppressive cell types in the multiple myeloma tumor microenvironment [52] as it reduces CD38 expression, but as treatment progresses, it also increases the resistance to treatment. Though this reduction is transient, it is regained in 3 to 6 months after the drug infusion. Another important concern is the reduction of CD38+ NK cells even after the first infusion. Though CD38 expression is low, NK cells retain their activity and proliferate normally $[88,89]$. In such a case, reinfusion of ex vivo expanded autologous NK cells can be used. 
TABLE 1: Currently active clinical trials with Daratumumab and Isatuximab.

\begin{tabular}{|c|c|c|c|c|}
\hline Tumor type & Drug & Patients & Title & Reference \\
\hline $\begin{array}{l}\text { Carcinoma, non-small- } \\
\text { cell lung }\end{array}$ & Daratumumab + Atezolizumab & 100 & $\begin{array}{l}\text { A study of Daratumumab in } \\
\text { combination with atezolizumab } \\
\text { compared with atezolizumab alone } \\
\text { in participants with previously } \\
\text { treated advanced or metastatic } \\
\text { nonsmall cell lung cancer }\end{array}$ & NCT03023423 \\
\hline Plasma cell myeloma & Daratumumab & 28 & $\begin{array}{l}\text { Daratumumab in treating patients } \\
\text { with multiple myeloma }\end{array}$ & NCT02944565 \\
\hline Multiple myeloma & Daratumumab & 50 & $\begin{array}{l}\text { Study of Daratumumab in multiple } \\
\text { myeloma (MM) patients in } \\
\text { >VGPR/MRD-positive. }\end{array}$ & NCT03992170 \\
\hline Multiple myeloma & Daratumumab + dexamethasone & 38 & $\begin{array}{l}\text { Efficacy of Daratumumab in } \\
\text { patients with relapsed/Refractory } \\
\text { myeloma with renal impairment }\end{array}$ & NCT03450057 \\
\hline $\begin{array}{l}\text { Relapsed/Refractory } \\
\text { multiple myeloma }\end{array}$ & $\begin{array}{l}\text { Nivolumab-Dara/nivolumab-Dara With low } \\
\text { dose of cyclophosphamide }\end{array}$ & 62 & $\begin{array}{l}\text { A phase } 2 \text { study of nivolumab } \\
\text { combined with Daratumumab with } \\
\text { or without low-dose } \\
\text { cyclophosphamide in relapsed/ } \\
\text { refractory multiple myeloma }\end{array}$ & \\
\hline Lymphoma & Daratumumab & 32 & $\begin{array}{l}\text { Phase } 2 \text { study to assess the clinical } \\
\text { efficacy and safety of Daratumumab } \\
\text { in patients with relapsed or } \\
\text { refractory natural killer/t cell } \\
\text { lymphoma, nasal type }\end{array}$ & NCT02927925 \\
\hline $\begin{array}{l}\text { Relapsed/Refractory } \\
\text { multiple myeloma }\end{array}$ & Daratumumab + ATRA & 60 & $\begin{array}{l}\text { A phase } 1 \text { and phase } 2 \text { study } \\
\text { of Daratumumab in combination } \\
\text { with all-trans retinoic acid in } \\
\text { relapsed/refractory multiple } \\
\text { myeloma }\end{array}$ & NCT02751255 \\
\hline $\begin{array}{l}\text { Pancreatic, non-small } \\
\text { cell lung or triple } \\
\text { negative breast cancers } \\
\text { (advanced or metastatic } \\
\text { solid tumors) }\end{array}$ & Nivolumab + Daratumumab & 120 & $\begin{array}{l}\text { Phase } 1 / 2 \text { study to evaluate the } \\
\text { safety and preliminary efficacy of } \\
\text { nivolumab combined } \\
\text { with Daratumumab in participants } \\
\text { with advanced or metastatic solid } \\
\text { tumors }\end{array}$ & NCT03098550 \\
\hline $\begin{array}{l}\text { Smoldering plasma cell } \\
\text { myeloma }\end{array}$ & Isatuximab & 62 & $\begin{array}{c}\text { Phase II single arm trial } \\
\text { of Isatuximab (SAR650984) in } \\
\text { patients with high risk smoldering } \\
\text { multiple myeloma }\end{array}$ & NCT02960555 \\
\hline Plasma cell myeloma & Isatuximab + cemiplimab & 109 & $\begin{array}{l}\text { A phase } 1 / 2 \text { study to evaluate safety, } \\
\text { pharmacokinetics and efficacy } \\
\text { of Isatuximab in combination with } \\
\text { cemiplimab in patients with } \\
\text { relapsed/refractory multiple } \\
\text { myeloma }\end{array}$ & NCT03194867 \\
\hline Multiple myeloma & Isatuximab + Bendamustine + prednisone & 37 & $\begin{array}{l}\text { A phase I/II trial of Isatuximab, } \\
\text { bendamustine, and prednisone in } \\
\text { pentarefractory multiple myeloma }\end{array}$ & NCT04083898 \\
\hline Lymphoma & Isatuximab + cemiplimab & 130 & $\begin{array}{c}\text { A phase } 1 / 2 \text { open-label, } \\
\text { multicenter, safety, preliminary } \\
\text { efficacy and pharmacokinetic (PK) } \\
\text { study of Isatuximab in combination } \\
\text { with other anticancer therapies in } \\
\text { participants with lymphoma }\end{array}$ & NCT03769181 \\
\hline
\end{tabular}


TABLE 1: Continued.

\begin{tabular}{|c|c|c|c|c|}
\hline Tumor type & Drug & Patients & Title & Reference \\
\hline $\begin{array}{l}\text { Prostate cancer non- } \\
\text { small cell lung cancer }\end{array}$ & Isatuximab + cemiplimab & 134 & $\begin{array}{c}\text { A phase } 1 / 2 \text { open-label, } \\
\text { multicenter, safety, preliminary } \\
\text { efficacy and pharmacokinetic (PK) } \\
\text { study of Isatuximab (SAR650984) } \\
\text { in combination with REGN2810, } \\
\text { or Isatuximab alone, in patients } \\
\text { with advanced malignancies }\end{array}$ & NCT03367819 \\
\hline Plasma cell myeloma & Isatuximab + lenalidomide + dexamethasone & 60 & $\begin{array}{l}\text { A phase } 1 \mathrm{~b} \text { study of SAR650984 } \\
\text { (Anti-CD38 mAb) in combination } \\
\text { with lenalidomide and } \\
\text { dexamethasone for the treatment of } \\
\text { relapsed or refractory multiple } \\
\text { myeloma }\end{array}$ & NCT01749969 \\
\hline Plasma cell myeloma & Isatuximab + lenalidomide + dexamethasone & 89 & $\begin{array}{l}\text { A phase } 1 \text { b study of SAR650984 } \\
\text { (isatuximab) in combination with } \\
\text { pomalidomide and dexamethasone } \\
\text { for the treatment of relapsed/ } \\
\text { refractory multiple myeloma }\end{array}$ & NCT02283775 \\
\hline
\end{tabular}

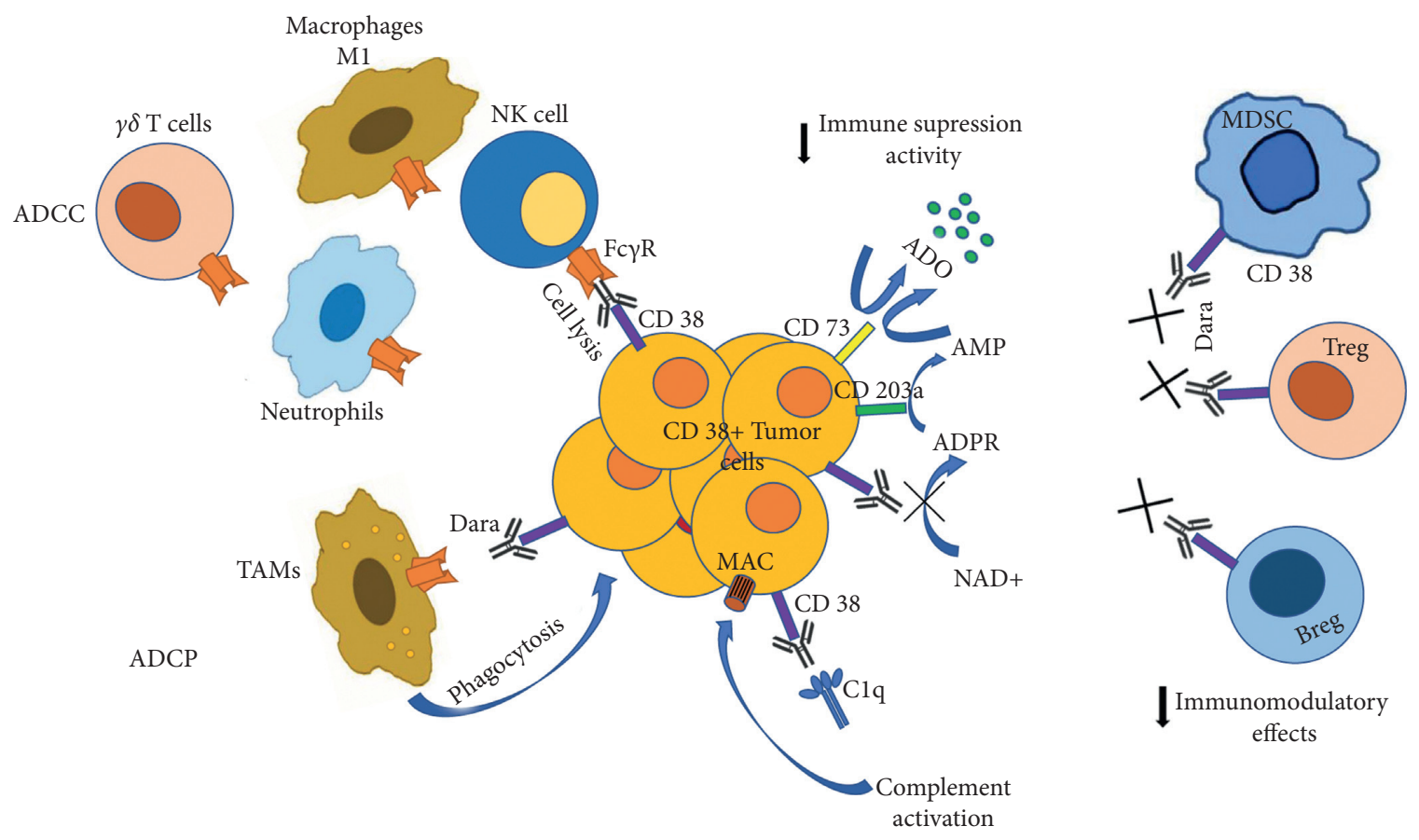

CDC

Figure 3: Dara mechanism of action. Dara exerts anticancer activity via Fc-dependent mechanism and immunomodulatory effects. Once bound to CD38 over cancer cells, Fc fragment of Dara allows engagement with Fc Receptors expressing effector cells, i.e., NK cells, T cells, neutrophils, and macrophages, leading to lysis (ADCC) or phagocytosis (ADCP) of the cancer cell. Engagement of Dara's Fc with C1q outcomes activation of compliment cascade and assembly of MAC complex over cancer cells and lysis (CDC). Dara's binding mask CD38 ectoenzymatic activity reduces adenosine production and causes eliminations of immune suppressive cell types (i.e.,Tregs, Bregs, MDSCs) that promotes T cell proliferation and effector functions (the figure is reproduced from the original figure published by Overdijk et al. [84] and distributed under the Creative Commons Attribution License).

To overcome Daratumumab mediated resistance, the use of new drugs such as a synthetic derivative of all-trans retinoic acid, Tamibarotene (a.k.a Am80), that upregulates CD38 or anti-CD38 antibodies with different mechanisms of action such as Isatuximab, Felzartamab, or Mezagitamab is recommended [90].

Isatuximab is an IgG1 monoclonal antibody that induces apoptosis of tumor cells and ADCC; it binds to a specific 
discontinuous epitope containing amino acids located opposite to the catalytic site of CD38, thus almost completely inhibiting cyclase activity in a dose-dependent manner [91]. Continuous Isatuximab treatment did not cause a reduction in CD38 receptor expression in H929, MM1S, OPM2, and RPMI-8226 multiple myeloma cell lines. Furthermore, Isatuximab treated cells did not show the clustering of CD38 in polar aggregates that lead to the release of CD38 in microvesicles, an effect that conduces to Daratumumab resistance [92]. Isatuximab substantiated great antitumor activity alone or in combination with dexamethasone and immunomodulatory imide drugs that include lenalidomide, pomalidomide, and iberdomide [93].

Mezagitamab (TAK-079) is a cytolytic IgG1 anti-CD38 monoclonal antibody, which effectively removes CD38+ $B$ cell lines by antibody-dependent or complement-dependent cytotoxicity [94].

Felzartamab (MOR202) is a Human Combinatorial Antibody Library derived human IgG1 anti-CD38 monoclonal antibody that, once attached, attracts natural killer cells, triggers $\mathrm{ADCC}$ and $\mathrm{ADCP}$ but not $\mathrm{CDC}$, and shows synergistically enhanced cytotoxicity with Bortezomib and Lenalidomide.

Other anti-CD38 agents are currently being evaluated. CAR-T/TCR-T, Multi-CAR-T, TAK-573, TAK-169, T-007, AMG 424, and GBR 1342 are in phase 1/2 clinical development, while others like HexaBody-CD38, CD38-ARM (KP1196, KP1237), TSK011010/CID103, STI-5171, AntiCD38/IGF-1 R bsAb scFV, Anti-CD38 SIFbod, CAR38MILs, CD38 DART, and Actinium-225 are in preclinical developmental stages. The significant number of potential candidates under development points to the importance of CD38 in the control of several malignancies.

Because of what has been mentioned, the efficacy of antiCD38 antibodies in many other cancers is being evaluated in preclinical and initial stages of clinical trials (Table 1).

\section{Conclusion}

CD38 has dual functions as an ectoenzyme and as a surface receptor that promotes migratory phenotypes and signaling cascades responsible for the activation and proliferation of various immune cells.

Both canonical and noncanonical pathways contribute to adenosine synthesis. However, is targeting CD38 alone sufficient to resolve ADO induced immunosuppression? CD38 expresses ubiquitously in immune populations like $\mathrm{T}$ cells, NK cells, and dendritic cells; therefore, targeting CD38 would reduce anti-inflammatory response and rejuvenate antitumor activity of immune cells. But the interconnecting links between CD38, CD39, and CD73 or with downstream adenosine receptors and the persistence of any compensatory mechanism available against CD38 depletion has to be further investigated. It is clear that the enzymatic and the surface receptor functions of CD38 are distinct from each other, and there is insufficient data available to justify which function of CD38 should be targeted for effective immune function restoration and hence, tumor elimination. Nevertheless, the development of anti-CD38 monoclonal antibodies has redefined the treatment landscape due to their ability to normalize immune cells function, thus triggering antibody-dependent cell-mediated cytotoxicity, complement-mediated cytotoxicity, antibody-dependent cellular phagocytosis of opsonized CD38+ cells, and direct apoptosis via $\mathrm{FC} \gamma$ receptor-mediated crosslinking. As it has been clearly stated by Morandi et al.[93]: CD38 is a receptor with modulatory functions on immune regulatory cell subsets that warrants deeper analysis.

\section{Conflicts of Interest}

The authors declare that none of them has conflicts of interest.

\section{Acknowledgments}

Special thanks are due to Dr. Leopoldo Santos Argumedo, Department of Molecular Biomedicine, Cinvestav, IPN, Mexico for his guidance. Sanyog Dwivedi is a PhD student from the Department of Molecular Biomedicine, CinvestavIPN, and receives a CONACYT CVU grant 871712. This work was supported by PAPIIT-UNAM grants IN221519 and IN218019.

\section{References}

[1] C. Donini, L. D’Ambrosio, G. Grignani, M. Aglietta, and D. Sangiolo, "Next generation immune-checkpoints for cancer therapy," Journal of Thoracic Disease, vol. 10, no. S13, pp. S1581-S1601, 2018.

[2] B. Zhang, "CD73 promotes tumor growth and metastasis," Oncoimmunology, vol. 1, no. 1, pp. 67-70, 2012.

[3] P. A. Beavis, J. Stagg, P. K. Darcy, and M. J. Smyth, "CD73: a potent suppressor of antitumor immune responses," Trends in Immunology, vol. 33, no. 5, pp. 231-237, 2012.

[4] S. Vigano, D. AlatzoglouM. Irving et al., "Targeting adenosine in cancer immunotherapy to enhance T-cell function," Frontier Immunology, vol. 10, p. 925, 2019.

[5] F. Morandi, D. Marimpietri, A. L. Horenstein et al., "Microvesicles released from multiple myeloma cells are equipped with ectoenzymes belonging to canonical and noncanonical adenosinergic pathways and produce adenosine from ATP and NAD," Oncoimmunology, vol. 7, no. 8, Article ID e1458809, 2018.

[6] S. Deaglio and S. C. Robson, "Ectonucleotidases as regulators of purinergic signaling in thrombosis, inflammation, and immunity," Advances in Pharmacology, vol. 61, pp. 301-332, 2011.

[7] A. L. Horenstein, A. Chillemi, G. Zaccarello et al., "A CD38/ CD203a/CD73 ectoenzymatic pathway independent of CD39 drives a novel adenosinergic loop in human T lymphocytes," Oncoimmunology, vol. 2, no. 9, Article ID e26246, 2013.

[8] T. A. Karakasheva, T. J. Waldron, E. Eruslanov et al., "CD38Expressing myeloid-derived suppressor cells promote tumor growth in a murine model of esophageal cancer," Cancer Research, vol. 75, no. 19, pp. 4074-4085, 2015.

[9] M. E. Moreno-García, L. N. López-Bojórques, A. Zentella, L. A. Humphries, D. J. Rawlings, and L. Santos-Argumedo, "CD38 signaling regulates B lymphocyte activation via a phospholipase C (PLC)- $\gamma 2$-Independent, protein kinase C, phosphatidylcholine-PLC, and phospholipase D-dependent 
signaling cascade," The Journal of Immunology, vol. 174, no. 5, pp. 2687-2695, 2005.

[10] B. P. Ramakers, K. E. Wever, M. Kox et al., "How systemic inflammation modulates adenosine metabolism and adenosine receptor expression in humans in vivo," Critical Care Medicine, vol. 40, no. 9, pp. 2609-2616, 2012.

[11] H. K. Eltzschig, M. V. Sitkovsky, and S. C. Robson, "Purinergic signaling during inflammation," New England Journal of Medicine, vol. 367, no. 24, pp. 2322-2333, 2012.

[12] I. C. van Rensburg and A. G. Loxton, "Killer (FASL regulatory) $\mathrm{B}$ cells are present during latent $\mathrm{TB}$ and are induced by BCG stimulation in participants with and without latent tuberculosis," Tuberculosis, vol. 108, pp. 114-117, 2018.

[13] E. Stocco, S. Barbon, M. Piccione et al., "Infrapatellar fat pad stem cells responsiveness to microenvironment in osteoarthritis: from morphology to function," Frontiers in Cell and Developmental Biology, vol. 7, p. 323, 2019.

[14] J. L. McKechnie, D. Beltrán, A.-M. M. Ferreira et al., "Mass cytometry analysis of the NK cell receptor-ligand repertoire reveals unique differences between dengue-infected children and adults," Immunohorizons, vol. 4, no. 10, pp. 634-647, 2020.

[15] M. A. Frassanito, F. Silvestris, N. Silvestris et al., "Fas/Fas ligand (FasL)-deregulated apoptosis and IL-6 insensitivity in highly malignant myeloma cells," Clinical \& Experimental Immunology, vol. 114, no. 2, pp. 179-188, 1998.

[16] Y. Gazitt, "TRAIL is a potent inducer of apoptosis in myeloma cells derived from multiple myeloma patients and is not cytotoxic to hematopoietic stem cells," Leukemia, vol. 13, no. 11, pp. 1817-1824, 1999.

[17] M. Gryko, K. Guzińska-Ustymowicz, A. Pryczynicz et al., "Correlation between Fas and FasL proteins expression in normal gastric mucosa and gastric cancer," Folia Histochemica et Cytobiologica, vol. 49, no. 1, pp. 142-147, 2011.

[18] H. Saito, S. Takaya, T. Osaki, and M. Ikeguchi, "Increased apoptosis and elevated Fas expression in circulating natural killer cells in gastric cancer patients," Gastric Cancer, vol. 16, no. 4, pp. 473-479, 2013.

[19] J. M. Konen, J. J. Fradette, and D. L. Gibbons, "The good, the bad and the unknown of CD38 in the metabolic microenvironment and immune cell functionality of solid tumors," Cells, vol. 9, no. 1, 2019.

[20] V. Quarona, G. Zaccarello, A. Chillemi et al., "CD38 and CD157: a long journey from activation markers to multifunctional molecules," Cytometry Part B: Clinical Cytometry, vol. 84B, no. 4, pp. 207-217, 2013.

[21] H. C. Lee, "Structure and enzymatic functions of human CD38," Molecular Medicine, vol. 12, no. 11-12, pp. 317-323, 2006.

[22] N. Gallay, L. Anani, A. Lopez et al., "The role of platelet/ endothelial cell adhesion molecule-1 (CD31) and CD38 antigens in marrow microenvironmental retention of acute myelogenous leukemia cells," Cancer Research, vol. 67, no. 18, pp. 8624-8632, 2007.

[23] S. A. Amici, N. A. Young, J. Narvaez-Miranda et al., "CD38 is robustly induced in human macrophages and monocytes in inflammatory conditions," Frontier Immunology, vol. 9, p. 1593, 2018.

[24] R. Aarhus, R. M. Graeff, D. M. Dickey, T. F. Walseth, and C. L. Hon, "ADP-ribosyl cyclase and CD38 catalyze the synthesis of a calcium-mobilizing metabolite from NADP+," Journal of Biological Chemistry, vol. 270, no. 51, pp. 3032730333, 1995.
[25] R. Bahri, A. Bollinger, T. Bollinger, Z. Orinska, and S. Bulfone-Paus, "Ectonucleotidase CD38 demarcates regulatory, memory-like CD8+ T cells with IFN- $\gamma$-mediated suppressor activities," PLoS One, vol. 7, no. 9, Article ID e45234, 2012.

[26] G. Fedele, L. Frasca, R. Palazzo, E. Ferrero, F. Malavasi, and C. M. Ausiello, "CD38 is expressed on human mature monocyte-derived dendritic cells and is functionally involved in CD83 expression and IL-12 induction," European Journal of Immunology, vol. 34, no. 5, pp. 1342-1350, 2004.

[27] R. Lande, F. Urbani, B. Di Carlo et al., "CD38 ligation plays a direct role in the induction of IL- $1 \beta$, IL- 6 , and IL- 10 secretion in resting human monocytes," Cellular Immunology, vol. 220, no. 1, pp. 30-38, 2002.

[28] X. Feng, L. Zhang, C. Acharya et al., "Targeting CD38 suppresses induction and function of Tregulatory cells to mitigate immunosuppression in multiple myeloma," Clinical Cancer Research, vol. 23, no. 15, pp. 4290-4300, 2017.

[29] Y. Yang, C. Li, T. Liu, X. Dai, and A. V. Bazhin, "Myeloidderived suppressor cells in tumors: from mechanisms to antigen specificity and microenvironmental regulation," Frontier Immunology, vol. 11, p. 1371, 2020.

[30] W. Wang, X. Yuan, H. Chen et al., "CD19+ CD24hiCD38hiBregs involved in downregulate helper $\mathrm{T}$ cells and upregulate regulatory T cells in gastric cancer," Oncotarget, vol. 6, no. 32, pp. 33486-33499, 2015.

[31] D. T. Patton, M. D. Wilson, W. C. Rowan, D. R. Soond, and K. Okkenhaug, "The PI3K p110 $\delta$ regulates expression of CD38 on regulatory T cells," PLoS One, vol. 6, no. 3, Article ID e17359, 2011.

[32] F. Flores-Borja, A. Bosma, D. Ng et al., "CD19+CD24hiCD38hi B cells maintain regulatory T cells while limiting TH1 and TH17 differentiation," Science Translational Medicine, vol. 5, no. 173, p. 173ra23, 2013.

[33] M. Domínguez-Pantoja, G. López-Herrera, H. RomeroRamírez et al., "CD38 protein deficiency induces autoimmune characteristics and its activation enhances IL-10 production by regulatory B cells," Scandinavian Journal of Immunology, vol. 87, no. 6, Article ID e12664, 2018.

[34] C. Chini, K. A. Hogan, G. M. Warner et al., "The NADase CD38 is induced by factors secreted from senescent cells providing a potential link between senescence and age-related cellular NAD+ decline," Biochemical and Biophysical Research Communications, vol. 513, no. 2, pp. 486-493, 2019.

[35] D. C. Hinshaw and L. A. Shevde, "The tumor microenvironment innately modulates cancer progression," Cancer Research, vol. 79, no. 18, pp. 4557-4566, 2019.

[36] L. Antonioli, C. Blandizzi, P. Pacher, and G. Haskó, "Immunity, inflammation and cancer: a leading role for adenosine," Nature Reviews Cancer, vol. 13, no. 12, pp. 842-857, 2013.

[37] E. Ferretti, A. L. Horenstein, C. Canzonetta, F. Costa, and F. Morandi, "Canonical and non-canonical adenosinergic pathways," Immunology Letters, vol. 205, pp. 25-30, 2019.

[38] A. Passarelli, M. Tucci, F. Mannavola, C. Felici, and F. Silvestris, "The metabolic milieu in melanoma: role of immune suppression by CD73/adenosine," Tumour Biology: The Journal of the International Society for Oncodevelopmental Biology and Medicine, vol. 42, no. 4, p. 1010428319837138 , 2019.

[39] I. Perrot, H.-A. Michaud, M. Giraudon-Paoli et al., "Blocking antibodies targeting the CD39/CD73 immunosuppressive pathway unleash immune responses in combination cancer therapies," Cell Reports, vol. 27, no. 8, pp. 2411-2425, 2019. 
[40] B. Ben Baruch, E. Blacher, E. Mantsur et al., "Stromal CD38 regulates outgrowth of primary melanoma and generation of spontaneous metastasis," Oncotarget, vol. 9, no. 61, pp. 31797-31811, 2018.

[41] A. Favia, M. Desideri, G. Gambara et al., "VEGF-induced neoangiogenesis is mediated by NAADP and two-pore channel-2-dependent Ca2+ signaling," Proceedings of the National Academy of Sciences, vol. 111, no. 44, pp. E4706E4715, 2014.

[42] A. Favia, I. Pafumi, M. Desideri et al., "NAADP-dependent Ca $(2+)$ signaling controls melanoma progression, metastatic dissemination and neoangiogenesis," Scientific Reports, vol. 6, p. 18925, 2016.

[43] A. S. Chung and N. Ferrara, "Developmental and pathological angiogenesis," Annual Review of Cell and Developmental Biology, vol. 27, no. 1, pp. 563-584, 2011.

[44] C. Pepper, T. T. Lin, G. Pratt et al., "Mcl-1 expression has in vitro and in vivo significance in chronic lymphocytic leukemia and is associated with other poor prognostic markers," Blood, vol. 112, no. 9, pp. 3807-3817, 2008.

[45] S. Chatterjee, A. Daenthanasanmak, P. Chakraborty et al., "CD38-NAD+Axis regulates immunotherapeutic anti-tumor T cell response," Cell Metabolism, vol. 27, no. 1, pp. 85-100, 2018.

[46] K. A. Hogan, C. C. S. Chini, and E. N. Chini, "The multifaceted ecto-enzyme CD38: roles in immunomodulation, cancer, aging, and metabolic diseases," Frontiers in Immunology, vol. 10, p. 1187, 2019.

[47] M. R. Fernandez and J. L. Cleveland, "Metabolic reprogramming via targeting CD38 NADase augments adoptive T cell therapy," Cell Metabolism, vol. 27, no. 1, pp. 3-5, 2018.

[48] N. W. C. J. van de Donk and S. Z. Usmani, "CD38 antibodies in multiple myeloma: mechanisms of action and modes of resistance," Frontiers in Immunology, vol. 9, p. 2134, 2018.

[49] S. Liao, S. Xiao, G. Zhu et al., "CD38 is highly expressed and affects the PI3K/Akt signaling pathway in cervical cancer," Oncology Reports, vol. 32, no. 6, pp. 2703-2709, 2014.

[50] S. Liao, L. Liang, C. Yue et al., "CD38 is involved in cell energy metabolism via activating the $\mathrm{PI} 3 \mathrm{~K} / \mathrm{AKT} / \mathrm{mTOR}$ signaling pathway in cervical cancer cells," International Journal of Oncology, vol. 57, no. 1, pp. 338-354, 2020.

[51] A. Lamanuzzi, I. Saltarella, V. Desantis et al., "Inhibition of mTOR complex 2 restrains tumor angiogenesis in multiple myeloma," Oncotarget, vol. 9, no. 29, pp. 20563-20577, 2018.

[52] J. Krejcik, T. Casneuf, I. S. Nijhof et al., "Daratumumab depletes CD38+ immune regulatory cells, promotes T-cell expansion, and skews T-cell repertoire in multiple myeloma," Blood, vol. 128, no. 3, pp. 384-394, 2016.

[53] L. Zhang, Y.-T. Tai, M. Ho et al., "Regulatory B cell-myeloma cell interaction confers immunosuppression and promotes their survival in the bone marrow milieu," Blood Cancer Journal, vol. 7, no. 3, p. e547, 2017.

[54] I. S. Nijhof, T. Casneuf, J. van Velzen et al., "CD38 expression and complement inhibitors affect response and resistance to daratumumab therapy in myeloma," Blood, vol. 128, no. 7 , pp. 959-970, 2016.

[55] E. García-Guerrero, R. Götz, S. Doose et al., "Upregulation of CD38 expression on multiple myeloma cells by novel HDAC6 inhibitors is a class effect and augments the efficacy of daratumumab," Leukemia, vol. 35, no. 1, pp. 201-214, 2021.

[56] E. García-Guerrero, T. Gogishvili, S. Danhof et al., "Panobinostat induces CD38 upregulation and augments the antimyeloma efficacy of daratumumab," Blood, vol. 129, no. 25, pp. 3386-3388, 2017.
[57] A. S. Laino, B. C. Betts, A. Veerapathran et al., "HDAC6 selective inhibition of melanoma patient T-cells augments antitumor characteristics," Journal for ImmunoTherapy of Cancer, vol. 7, no. 1, p. 33, 2019.

[58] V. Kotla, S. Goel, S. Nischal et al., "Mechanism of action of lenalidomide in hematological malignancies," Journal of Hematology \& Oncology, vol. 2, no. 1, p. 36, 2009.

[59] Y. H. Huang, M. R. Vakili, O. Molavi et al., "Decoration of anti-CD38 on nanoparticles carrying a STAT3 inhibitor can improve the therapeutic efficacy against myeloma," Cancers (Basel), vol. 11, no. 2, 2019.

[60] S. Liao, S. Xiao, H. Chen et al., "CD38 enhances the proliferation and inhibits the apoptosis of cervical cancer cells by affecting the mitochondria functions," Molecular Carcinogenesis, vol. 56, no. 10, pp. 2245-2257, 2017.

[61] D. S. Chen and I. Mellman, "Elements of cancer immunity and the cancer-immune set point," Nature, vol. 541, no. 7637, pp. 321-330, 2017.

[62] P. Bhat, G. Leggatt, N. Waterhouse, and I. H. Frazer, "Interferon- $\gamma$ derived from cytotoxic lymphocytes directly enhances their motility and cytotoxicity," Cell Death and Disease, vol. 8, no. 6, Article ID e2836, 2017.

[63] J. H. Lam, H. H. M. Ng, C. J. Lim et al., "Expression of CD38 on macrophages predicts improved prognosis in hepatocellular carcinoma," Frontiers Immunology, vol. 10, p. 2093, 2019.

[64] M. Garnelo, A. Tan, Z. Her et al., "Interaction between tumour-infiltrating B cells and T cells controls the progression of hepatocellular carcinoma," Gut, vol. 66, no. 2, pp. 342-351, 2017.

[65] M.-T. Zilber, S. Gregory, R. Mallone et al., "CD38 expressed on human monocytes: a coaccessory molecule in the superantigen-induced proliferation," Proceedings of the National Academy of Sciences, vol. 97, no. 6, pp. 2840-2845, 2000.

[66] H. H. M. Ng, R. Y. Lee, S. Goh et al., "Immunohistochemical scoring of CD38 in the tumor microenvironment predicts responsiveness to anti-PD-1/PD-L1 immunotherapy in hepatocellular carcinoma," Journal for ImmunoTherapy of Cancer, vol. 8, no. 2, 2020.

[67] X. Bu, J. Kato, J. A. Hong et al., "CD38 knockout suppresses tumorigenesis in mice and clonogenic growth of human lung cancer cells," Carcinogenesis, vol. 39, no. 2, pp. 242-251, 2018.

[68] E. B. Garon, N. A. Rizvi, R. Hui et al., "Pembrolizumab for the treatment of non-small-cell lung cancer," New England Journal of Medicine, vol. 372, no. 21, pp. 2018-2028, 2015.

[69] A. O. Kamphorst, R. N. Pillai, S. Yang et al., "Proliferation of PD-1+ CD8 T cells in peripheral blood after PD-1-targeted therapy in lung cancer patients," Proceedings of the National Academy of Sciences, vol. 114, no. 19, pp. 4993-4998, 2017.

[70] E. Tassi, G. Grazia, C. Vegetti et al., "Early effector $\mathrm{T}$ lymphocytes coexpress multiple inhibitory receptors in primary non-small cell lung cancer," Cancer Research, vol. 77, no. 4, pp. 851-861, 2017.

[71] E. Blacher, B. Ben Baruch, A. Levy et al., "Inhibition of glioma progression by a newly discovered CD38 inhibitor," International Journal of Cancer, vol. 136, no. 6, pp. 1422-1433, 2015.

[72] A. Levy, E. Blacher, H. Vaknine, F. E. Lund, R. Stein, and L. Mayo, "CD38 deficiency in the tumor microenvironment attenuates glioma progression and modulates features of tumor-associated microglia/macrophages," Neuro-Oncology, vol. 14, no. 8, pp. 1037-1049, 2012.

[73] M. L. Goodenberger and R. B. Jenkins, "Genetics of adult glioma," Cancer Genetics, vol. 205, no. 12, pp. 613-621, 2012. 
[74] F. B. Furnari, T. Fenton, R. M. Bachoo et al., "Malignant astrocytic glioma: genetics, biology, and paths to treatment," Genes \& Development, vol. 21, no. 21, pp. 2683-2710, 2007.

[75] B. Badie and J. Schartner, "Role of microglia in glioma biology," Microscopy Research and Technique, vol. 54, no. 2, pp. 106-113, 2001.

[76] I. Yang, S. J. Han, G. Kaur, C. Crane, and A. T. Parsa, “The role of microglia in central nervous system immunity and glioma immunology," Journal of Clinical Neuroscience, vol. 17, no. 1, pp. 6-10, 2010.

[77] A. Levy, A. Bercovich-Kinori, A. G. Alexandrovich et al., "CD38 facilitates recovery from traumatic brain injury," Journal of Neurotrauma, vol. 26, no. 9, pp. 1521-1533, 2009.

[78] L. Mayo, J. Jacob-Hirsch, N. Amariglio et al., "Dual role of CD38 in microglial activation and activation-induced cell death," The Journal of Immunology, vol. 181, no. 1, pp. 92-103, 2008.

[79] M. R. Albertini, "The age of enlightenment in melanoma immunotherapy," Journal for ImmunoTherapy of Cancer, vol. 6 , no. 1, p. 80, 2018.

[80] F. Spinella, V. Caprara, R. Cianfrocca et al., "The interplay between hypoxia, endothelial and melanoma cells regulates vascularization and cell motility through endothelin-1 and vascular endothelial growth factor," Carcinogenesis, vol. 35, no. 4, pp. 840-848, 2014.

[81] F. Morandi, B. Morandi, A. L. Horenstein et al., "A noncanonical adenosinergic pathway led by CD38 in human melanoma cells induces suppression of T cell proliferation," Oncotarget, vol. 6, no. 28, pp. 25602-25618, 2015.

[82] M. H. Z. Guang, A. McCann, G. Bianchi et al., "Overcoming multiple myeloma drug resistance in the era of cancer “omics", Leukemia \& Lymphoma, vol. 59, no. 3, pp. 542-561, 2018.

[83] S. Z. Usmani, B. M. Weiss, T. Plesner et al., "Clinical efficacy of daratumumab monotherapy in patients with heavily pretreated relapsed or refractory multiple myeloma," Blood, vol. 128, no. 1, pp. 37-44, 2016.

[84] M. B. Overdijk, S. Verploegen, M. Bögels et al., "Antibodymediated phagocytosis contributes to the antitumor activity of the therapeutic antibody daratumumab in lymphoma and multiple myeloma," MAbs, vol. 7, no. 2, pp. 311-320, 2015.

[85] I. S. Nijhof, R. W. J. Groen, H. M. Lokhorst et al., "Upregulation of CD38 expression on multiple myeloma cells by alltrans retinoic acid improves the efficacy of daratumumab," Leukemia, vol. 29, no. 10, pp. 2039-2049, 2015.

[86] M.-V. Mateos, M. A. Dimopoulos, M. Cavo et al., "Daratumumab plus bortezomib, melphalan, and prednisone for untreated myeloma," New England Journal of Medicine, vol. 378, no. 6, pp. 518-528, 2018.

[87] P. Moreau, M. Attal, C. Hulin et al., "Bortezomib, thalidomide, and dexamethasone with or without daratumumab before and after autologous stem-cell transplantation for newly diagnosed multiple myeloma (CASSIOPEIA): a randomised, open-label, phase 3 study," Lancet (London, England), vol. 394, no. 10192, pp. 29-38, 2019.

[88] T. Casneuf, X. S. Xu, H. C. Adams et al., "Effects of daratumumab on natural killer cells and impact on clinical outcomes in relapsed or refractory multiple myeloma," Blood Advances, vol. 1, no. 23, pp. 2105-2114, 2017.

[89] Y. Wang, Y. Zhang, T. Hughes et al., "Fratricide of NK cells in daratumumab therapy for multiple myeloma overcome by ex vivo-expanded autologous NK cells," Clinical Cancer Research, vol. 24, no. 16, pp. 4006-4017, 2018.
[90] K. A. Frerichs, N. A. Nagy, P. L. Lindenbergh et al., "CD38targeting antibodies in multiple myeloma: mechanisms of action and clinical experience," Expert Review of Clinical Immunology, vol. 14, no. 3, pp. 197-206, 2018.

[91] J. Deckert, M. C. Wetzel, L. M. Bartle et al., "SAR650984, A novel humanized CD38-targeting antibody, demonstrates potent antitumor activity in models of multiple myeloma and other CD38+ hematologic malignancies," Clinical Cancer Research, vol. 20, no. 17, pp. 4574-4583, 2014.

[92] L. Moreno, C. Perez, A. Zabaleta et al., "The mechanism of action of the anti-CD38 monoclonal antibody Isatuximab in multiple myeloma," Clinical Cancer Research, vol. 25, no. 10, pp. 3176-3187, 2019.

[93] F. Morandi, I. Airoldi, D. Marimpietri, C. Bracci, A. C. Faini, and R. Gramignoli, "CD38, a receptor with multifunctional activities: from modulatory functions on regulatory cell subsets and extracellular vesicles, to a target for therapeutic strategies," Cells, vol. 8, no. 12, 2019.

[94] W. Korver, M. Carsillo, J. Yuan et al., "A reduction in B, T, and natural killer cells expressing CD38 by TAK-079 inhibits the induction and progression of collagen-induced arthritis in cynomolgus monkeys," Journal of Pharmacology and Experimental Therapeutics, vol. 370, no. 2, pp. 182-196, 2019. 